\title{
THE UNITARY REPRESENTATION THEORY OF GL $(n)$ OF AN INFINITE DISCRETE FIELD
}

\author{
BY \\ ROGER E. HOWE ${ }^{\dagger, a}$ AND JONATHAN ROSENBERG ${ }^{\dagger, b}$ \\ 'Department of Mathematics, Yale University, New Haven, CT 06520, USA; and \\ 'Department of Mathematics, University of Maryland, College Park, MD 20742, USA
}

\begin{abstract}
If $K$ is an infinite field and if $G=\mathrm{GL}(n, K)$ with the discrete topology, then all principal-series representations of $G$ are irreducible, and any two such with the same central character $\psi$ are weakly equivalent to one another and to the $\psi$-regular representation. In addition, every irreducible unitary representation of $G$ which is not one-dimensional weakly contains a representation of the principal series. We deduce that every maximal ideal of $C^{*}(G)$ is either of codimension 1 or else a kernel of a principal-series representation. In particular, except in the exceptional case where $K$ is an infinite algebraic extension of a finite field, the reduced $C^{*}$-algebra of $\operatorname{PGL}(n, K)$ is simple, as was also shown by de la Harpe in many cases.
\end{abstract}

\section{Introduction, notation and statement of results}

Suppose $K$ is an infinite field, $K^{\times}$is its multiplicative group, $n \geqq 2$, and $G=\mathrm{GL}(n, K)$. We give all of these the discrete topology. Also we usually think of $K$ just as an additive group, and let $\hat{K}$ denote its Pontryagin dual group, which is compact. We shall consider in this paper the unitary representation theory of the group $G$. There are many motivations for studying this topic. One is that $G$ is one of the few highly non-commutative infinite discrete groups about whose unitary representations one can say very much. (Other known

† Partially supported by NSF Grant DMS-85-06130. It is a pleasure also to acknowledge the hospitality of the Institute for Advanced Studies, The Hebrew University of Jerusalem, 91904 Jerusalem, Israel, from January to August, 1988.

\# Partially supported by NSF Grants DMS-84-00900 and DMS-87-00551. Much of this work was done while visiting at, and partially supported by, the Department of Mathematics and Computer Science, Bar-Ilan University, 52100 Ramat Gan, Israel.

Received August 25, 1988 
examples tend to be amenable, such as the infinite symmetric group, studied by Thoma [10], and finitely generated torsion-free nilpotent groups [4]. On the other hand, $G$ is amenable only in one special case - see Proposition 9 below.) A second motivation is that when $K$ is a number field (the case of primary interest to us), $G$ embeds as a lattice subgroup of $G L\left(n, \mathbf{A}_{K}\right)$, and the study of the interaction between the unitary representations of these two groups might encode some number-theoretic information. It is also possible that the study of the unitary representation theory of $G$ should also give some clue of what one should expect for arithmetic groups such as $\mathrm{GL}\left(n, \mathcal{O}_{K}\right)$, e.g., $\mathrm{GL}(n, \mathbf{Z})$. (Nevertheless, there are substantial differences between $\mathrm{GL}(n, K)$ and $\mathrm{GL}(n, \mathbf{Z})$ - for example, as the former is not finitely generated, it can't have Kazhdan's property $T$ [8], though the latter does for $n \geqq 3$.)

We let $B$ denote the Borel subgroup of $G$ consisting of upper-triangular matrices, so that $B=A \ltimes N$, where $A \cong\left(K^{\times}\right)^{n}$ is the group of invertible diagonal matrices and $N$ is the group of upper-triangular matrices with 1's on the diagonal. Let $W$ denote the group of permutation matrices in $G$, the matrices with exactly one 1 in each row and column and with 0 's elsewhere. The group $W$, which is isomorphic to $S_{n}$, may be identified with the Weyl group of $A$, and by Bruhat decomposition, $G=\amalg_{w \in W} B w B$. (See, for instance, $28.3,34.5$ and 35.1 in [6], though for GL $(n)$ this is much more elementary, and follows from basic linear algebra as in [7], 3.7.) We also denote by $Z$ the group of scalar matrices, which is the center of $G$; note that $Z \cong K^{\times}$. All these groups are given the discrete topology.

All group representations to be considered in this paper are unitary representations on Hilbert spaces. As usual, $\hat{G}$ denotes the set of equivalence classes of irreducible representations of $G$, and $C^{*}(G)$ denotes the group $C^{*}$ algebra of $G$, the completion of $C G$ in the greatest $C^{*}$-norm. Any unitary representation $\pi$ of $G$ canonically extends to a *-representation of $C^{*}(G)$, and when we refer to the kernel of $\pi$, $\operatorname{ker} \pi$, we usually mean the kernel in $C^{*}(G)$, rather than the kernel in $G$ (which is usually uninteresting, since $G$ is close to being simple). We denote by Prim $G$ the primitive ideal space of $C^{*}(G)$, with the Jacobson topology. A representation $\pi$ is said to be weakly contained in a representation $\sigma$ iff $\operatorname{ker} \pi \supseteq \operatorname{ker} \sigma$ in $C^{*}(G)$, or equivalently (by Fell's Theorem - [2] or $[1,18.1 .4])$, if any matrix coefficient of $\pi$ may be approximated pointwise by matrix coefficients of a multiple of $\sigma$. Representations are weakly equivalent if each is weakly contained in the other, i.e., if they have the same kernel in $C^{*}(G)$. Since $G$ is not type I (the only discrete groups that are type I are those with an abelian subgroup of finite index [11]), the natural surjection 
$\hat{G} \rightarrow$ Prim $G$ (sending $\pi$ to $\operatorname{ker} \pi$ ) is not one-to-one, and for $K$ countable, $\hat{G}$ is not even countably separated as a Borel space $[1,9.5 .6]$. Thus we cannot realistically expect to do any better than to classify irreducible representations up to weak equivalence, i.e., to compute Prim $G$. One of us accomplished this in [4] for finitely generated torsion-free nilpotent groups, and the answer in that case turned out to parallel to some extent the Kirillov theory of unitary representations of nilpotent Lie groups. Thus one might expect the classification of Prim $G$ to reflect some of what is known about unitary representations of $\mathrm{GL}(n)$ over finite or local fields. However, as we shall see, the theory is substantially different.

For every $\pi \in \hat{G},\left.\pi\right|_{z}$ must be a multiple of a character $\psi$, called the central character of $\pi$. In this way, one obtains a natural surjection Prim $G \rightarrow$ $\hat{Z} \cong\left(K^{\times}\right)^{\wedge}$. For $\psi \in \hat{Z}$, the fiber is denoted $\operatorname{Prim}(G, \psi)$ and corresponds to a quotient $C^{*}(G, \psi)$ of $C^{*}(G)$. One obvious representation of this algebra is

$$
\lambda_{\psi}=\operatorname{Ind}_{z \nmid G} \psi
$$

where Ind stands for unitary induction, which we call the $\psi$-regular representation of $G$. Since $G / Z$ is an infinite-conjugacy-class group, this is a $\mathrm{II}_{1}$ factor representation. We denote by $C_{r}^{*}(G, \psi)$ the $C^{*}$-algebra $\lambda_{\psi}\left(C^{*}(G, \psi)\right)$. This algebra is prime, and in fact it will turn out that it is primitive (Theorem 4 below - of course, the result is automatic by $C^{*}$-algebra theory if $K$ (and hence $G$ ) is countable [1, 3.9.1.c]) and almost always simple (note part (c) of that same theorem).

It is easy to specify certain special irreducible representations of $G$ which will play an important role in what follows. First, if $\varphi \in\left(K^{\times}\right)^{\wedge}, \varphi \circ$ det defines a one-dimensional irreducible unitary representation of $G$, with central character $\varphi^{n}$. Since $G_{a b}$ is isomorphic to $K^{\times}$via the determinant map [7, pp. 376-377], all one-dimensional unitary representations are of this form. Also note that if $\mu_{n}^{K}$ denotes the group of $n$-th roots of unity in $K$, which of course has order at most $n$, then we have the exact sequence of discrete abelian groups

$$
1 \rightarrow \mu_{n}^{K} \rightarrow K^{\times} \stackrel{\bullet}{\rightarrow} K^{\times} \rightarrow K^{\times} /\left\{n \text {-th powers in } K^{\times}\right\} \rightarrow 1,
$$

and thus the dual exact sequence of compact groups

$$
1 \rightarrow\left(K^{\times} /\left\{n \text {-th powers in } K^{\times}\right\}\right)^{\wedge} \rightarrow\left(K^{\times}\right)^{\wedge} \stackrel{\bullet}{\rightarrow}\left(K^{\times}\right)^{\wedge} \rightarrow\left(\mu_{n}^{K}\right)^{\wedge} \rightarrow 1 .
$$

If follows immediately that for $\psi \in\left(K^{\times}\right)^{\wedge}, \psi$ can be written as $\varphi^{n}$, for some 
$\varphi \in\left(K^{\times}\right)^{\wedge}$, if and only if $\psi$ vanishes on $\mu_{n}^{K}$. When this is the case, we also see that the possible $\varphi$ 's are parameterized (non-canonically) by the character group of $K^{\times} /\left\{n\right.$-th powers in $\left.K^{\times}\right\}$. In particular, there is only one if $K$ is algebraically closed, or if $n$ is a power of the characteristic and $K$ is perfect.

The other basic representations of $G$ are the principal series. If $\psi_{1}, \ldots, \psi_{n} \in$ $\left(K^{\times}\right)^{\wedge}$, then $\left(\psi_{1}, \ldots, \psi_{n}\right)$ may be viewed as a character of $A$ and hence of $B$ (by extending to be trivial on $N$ ). The corresponding principal-series representation of $G$ is defined to be $\operatorname{Ind}_{B \nmid G}\left(\psi_{1}, \ldots, \psi_{n}\right)$. Its central character is easily computed to be $\psi=\psi_{1} \psi_{2} \cdots \psi_{n}$. Similarly, one can define other series of representations, called degenerate principal series, attached to standard parabolic subgroups other than $B$. Given positive integers $m_{1}, \ldots, m_{r}$ with $m_{1}+\cdots+m_{r}=n$, one can define the standard parabolic subgroup of blockupper-triangular matrices

$$
\begin{aligned}
P_{\left(m_{1}, \ldots, m_{r}\right)} & =\left\{\left(\begin{array}{c|c|c}
* & * & * \\
\hline 0 & * & * \\
\hline 0 & 0 & \ddots
\end{array}\right) \begin{array}{c}
\} m_{1} \\
\} m_{2} \\
\vdots
\end{array}\right\} \\
& =\left(\mathrm{GL}\left(m_{1}, K\right) \times \cdots \times \mathrm{GL}\left(m_{r}, K\right)\right) \times N_{\left(m_{1}, \ldots, m_{r}\right)} .
\end{aligned}
$$

where

$$
N_{\left(m_{1}, \ldots, m_{l}\right)}=\left\{\left(\begin{array}{c|c|c}
1 & * & * \\
\hline 0 & 1 & * \\
\hline 0 & 0 & \ddots
\end{array}\right) \begin{array}{c}
m_{1} \\
\} m_{2} \\
\vdots
\end{array}\right\}
$$

Then given $\psi_{1}, \ldots, \psi_{r} \in\left(K^{\times}\right)^{\wedge}$, one can form the character

$$
\left(\psi_{1} \circ \mathrm{det}\right) \otimes \cdots \otimes\left(\psi_{r} \circ \mathrm{det}\right)
$$

of $\mathrm{GL}\left(m_{1}, K\right) \times \cdots \times \mathrm{GL}\left(m_{r}, K\right)$. This can be extended to $P_{\left(m_{1}, \ldots, m_{r}\right)}$ by making it trivial on $N_{\left(m_{1}, \ldots, m_{t}\right)}$ and inducing unitarily to $G$ gives a representation of the degenerate principal series for our chosen parabolic subgroup.

We now have established the notation necessary to state our main results. As the first of these is rather trivial, it does not seem appropriate to call it a theorem.

Proposition 1. Any principal-series representation of $G$, and in fact any degenerate-principal-series representation of $G$, is irreducible. 
Theorem 2. Any unitary representation of $G$ which is non-trivial on the commutator subgroup $\operatorname{SL}(n, K)$ weakly contains a representation of the principal series.

THEOREM 3. For any $\psi \in\left(K^{\times}\right)^{\wedge}$, any principal-series representation with this central character is weakly equivalent to $\lambda_{\psi}$. Hence any two such principalseries representations are weakly equivalent to each other.

By putting all these results together, we obtain the following:

THEOREM 4. (a) For each $\psi \in Z^{\wedge}, C^{*}(G, \psi)$ has at most one maximal ideal of infinite codimension, namely, $\operatorname{ker} \lambda_{\psi}$. (It can fail to be maximal only when $K$ is an infinite algebraic extension of a finite field.) If $\psi$ is non-trivial on $\mu_{n}^{K}$, this is the unique maximal ideal. Otherwise the other maximal ideals are the kernels of the one-dimensional representations $\varphi \circ$ det with $\varphi^{n}=\psi$.

(b) Except when $K$ is an infinite algebraic extension of a finite field, $C_{r}^{*}(G, \psi)$ is simple for each $\psi$. In particular, $C_{r}^{*}(\operatorname{PGL}(n, K))$ is simple.

(c) If $K$ is an infinite algebraic extension of $\mathbf{F}_{p}$, then $C^{*}(G, \psi)$ is primitive for each $\psi$. The only primitive ideals of this algebra, other than $\{0\}$, are of codimension 1 . Hence if $\psi$ does not vanish on $\mu_{n}^{K}, C^{*}(G, \psi)$ is simple.

Note that the simplicity of $C_{r}^{*}(\operatorname{PGL}(n, K))$ for fields $K$ of characteristic 0 was conjectured by de la Harpe and proved for $n \leqq 3$ [3- but see the note "added in proof" on p. 253]. It is interesting to see that the same holds for most fields of characteristic $p$.

Some of the results of this paper were obtained several years ago and announced at a meeting of the American Mathematical Society in Baltimore in 1986 [5]. However, we have simplified many of the proofs in the meantime.

\section{Proofs of the theorems}

We proceed now to prove all the theorems stated above. Proposition 1 follows easily from the following version of the Frobenius reciprocity theorem, due to Mackey [9, Theorem 4']. Mackey assumes separability of the Hilbert spaces, which is never really used in the proof. Since the result is very easy, we include a (slightly different) proof for completeness.

Theorem 5 (Mackey [9]). Let $G$ be a discrete group, $P$ a subgroup, $\pi$ a unitary representation of $P$ on a Hilbert space $H_{\pi}$, and $\sigma$ a unitary representation of $G$ on a Hilbert space $H_{\sigma}$. Then the map 


$$
T \mapsto S, \quad \text { where } S \varphi=(T \varphi)(e),
$$

induces an injection

$$
\operatorname{Hom}_{G}\left(\sigma, \operatorname{Ind}_{P \uparrow G} \pi\right) \hookrightarrow \operatorname{Hom}_{P}\left(\left.\sigma\right|_{P}, \pi\right) .
$$

Proof. Recall that $\operatorname{Ind}_{P \uparrow G} \pi$ acts on the Hilbert space

$$
\begin{aligned}
& V=\left\{f: G \rightarrow H_{\pi} \mid f(g b)=\pi(b)^{-1} f(g) \text { for } g \in G, b \in P ;\right. \\
&\left.(\dot{g} \mapsto\|f(g)\|) \in L^{2}(G / P)\right\} .
\end{aligned}
$$

Since $G$ is discrete, the point evaluation functionals $f \mapsto f(g)$ are bounded on $V$, so it is clear that the map $T \mapsto S$ as defined is bounded. A simple calculation shows that this map sends a $G$-intertwining operator $T: H_{\sigma} \rightarrow V$ to a $P$-intertwining operator $S: H_{\sigma} \rightarrow H_{\pi}$. The map is an injection, since if $S \equiv 0$, then for any $v \in H_{\sigma}$ and $g \in G$, we have

$$
0 \equiv S\left(\sigma(g)^{-1} v\right)=T\left(\sigma\left(g^{-1}\right) v\right)(e)=\sigma\left(g^{-1}\right)(T v)(e)=(T v)(g),
$$

hence $T v \equiv 0$.

Remark. When $G$ is infinite, the map of the theorem is usually not a surjection.

Corollary (Proposition 1). Let $K$ be an infinite discrete field, $G=$ $\mathrm{GL}(n, K)$, and $P=P_{\left(m_{1}, \ldots, m_{r}\right)}$ some standard parabolic subgoup of $G$. Then for any one-dimensional unitary character $\varphi$ of $P, \operatorname{Ind}_{P \uparrow G} \varphi$ is irreducible.

Proof. By Theorem 5, $\operatorname{Hom}_{G}\left(\operatorname{Ind}_{P \nmid G} \varphi, \operatorname{Ind}_{P \nmid G} \varphi\right) \hookrightarrow \operatorname{Hom}_{P}\left(\operatorname{Ind}_{P \nmid G} \varphi, \varphi\right)$. We claim the latter is one-dimensional. Indeed, by Bruhat decomposition, $G$ is a finite union of $P-P$ double cosets $P w_{i} P$, with $w_{1}=1$ and $w_{i} \notin P$ for $i>1$. If we write $P=M N$, where $N$ is the unipotent radical of $P$, then the functions in the Hilbert space $V$ of $\operatorname{Ind}_{P \uparrow G} \varphi$ which are supported on $P w_{i} P$ are an $N$ invariant subspace which does not contain the trivial representation of $N$ for $i>1$ (since $K$ is infinite). Thus

$$
\operatorname{dim} \operatorname{Hom}_{P}\left(\operatorname{Ind}_{P \uparrow G} \varphi, \varphi\right) \leqq \operatorname{dim} \operatorname{Hom}_{N}\left(\operatorname{Ind}_{P \uparrow G} \varphi, \varphi\right)=1 .
$$

The proofs of the main theorems, Theorems $2-4$, will be fairly straightforward once we have proved a few preliminary results. The first of these is valid for arbitrary discrete groups and will be used many times below.

LEMMA 6. Let $L$ be any discrete group and $M$ any subgroup. Suppose $\pi$ is a unitary representation of $L$ whose restriction to $M$ weakly contains some repre- 
sentation $\rho$ of $M$. Then $\pi$ weakly contains a representation $\sigma$ of $L$ such that $\left.\sigma\right|_{M}$ contains $\rho$ (literally, not just weakly). If $\rho$ is irreducible, $\sigma$ can be chosen irreducible.

Proof. It is obviously no loss of generality to assume $\rho$ is cyclic with some unit cyclic vector $\xi$, since we can deal with any cyclic component separately. By assumption, there are normalized matrix coefficients $\varphi_{\alpha}$ of multiples of $\pi$, whose restrictions to $M$ constitute a net converging to the coefficient $m \mapsto\langle\rho(m) \xi, \xi\rangle$ of $\rho$. Since the space of normalized positive-definite functions on $L$ is compact in the topology of pointwise convergence, there is a subnet converging to a positive-definite function $\varphi$. Then $\varphi$ can be realized as $\langle\sigma(\cdot) \eta, \eta\rangle$, for some representation $\sigma$ of $L$ and unit cyclic vector $\eta$. Clearly $\sigma$ has the desired property. If $\rho$ is irreducible, then there are irreducible representations of $L$ whose restrictions to $M$ contain $\rho$, and we can take $\sigma$ to be one of these.

The proof of Theorem 2 requires two more preliminaries. The first gives us a tool to handle representations of certain subgroups of $G$, and the second gives a way of "recognizing" representations of the principal series.

Proposition 7. Consider the action of $G=\mathrm{GL}(n, K)$ on $\left(K^{n}\right)^{\wedge}$, dual to the usual action on $K^{n}$.

(i) If $\psi \neq 1$ in $\left(K^{n}\right)^{\wedge}$, then the $G$-orbit of $\psi$ is dense in $\left(K^{n}\right)^{\wedge}$.

(ii) If $K$ is countable, then the set of $\psi$ with trivial stabilizer is dense $G_{\delta}$ of full Haar measure. If $n=1$, then whether $K$ is countable or not, $G$ acts freely on $\hat{K}-\{1\}$.

Proof. (i) Write $\psi=\left(\psi_{1}, \ldots, \psi_{n}\right)$ with $\psi_{i} \in \hat{K}$. If $\psi \neq 1$, then transforming by an element of $G$ if necessary, we can asume $\psi_{i} \neq 1$ for all $i$. Then considering the action of the diagonal subgroup $A$ of $G$ shows it suffices to prove the result in the case $n=1$.

So take $n=1$. For $a \in K^{\times}=\mathrm{GL}(1, K)$, let $\psi_{a}=a^{-1} \cdot \psi$, so

$$
\psi_{a}(x)=\psi(a x), \quad x \in K .
$$

Note that $\psi_{a} \psi_{b}=\psi_{a+b}$, so if we let $\psi_{0}=1_{K}$, we see that

$$
H=\left\{\psi_{a}: a \in K\right\}=K^{\times} \cdot \psi \cup\left\{1_{K}\right\}
$$

is a subgroup of $\hat{K}$. If $K^{\times} \cdot \psi$ were not dense in $\hat{K}$, then $\bar{H}$ would be a proper closed subgroup, and by Pontryagin duality there would be some $0 \neq x \in K$ annihilating $H$. This would mean 


$$
\psi(a x)=1 \quad \text { for all } a \in K,
$$

hence $\psi(y)=1$ for all $y \in K$, i.e., $\psi=1_{K}$, a contradiction. So $K^{\times} \cdot \psi$ is dense.

(ii) For $g \in G$ and $\psi \in\left(K^{n}\right)^{\wedge}, g \cdot \psi=\psi$ if and only if

$$
(g-1)\left(K^{n}\right) \subseteq \operatorname{ker} \psi .
$$

Here 1 denotes the identity element of $G$. If $g \neq 1$, then $(g-1)\left(K^{n}\right)$ is a non-zero linear subspace of $K^{n}$. If $n=1$, this means it is everything, and (*) forces $\psi=1_{K}$. In general, the set $\left(\left(K^{n}\right)^{\wedge}\right)^{8}$ of $g$-fixed points in $\left(K^{n}\right)^{\wedge}$ is the annihilator of this subspace, and so is a closed subgroup with void interior and Haar measure 0 . If $K$ is countable, then so is $G$, and the set of $\psi$ 's with trivial stabilizer is the intersection of countably many dense open sets of full Haar measure. Density follows by the Baire category theorem.

LEMMA 8. Let $\pi$ be an irreducible unitary representation of $G=\mathrm{GL}(n, K)$, and suppose the representation space $\pi$ contains a non-zero B-eigenvector $\xi$ for a character $\psi=\left(\psi_{1}, \ldots, \psi_{n}\right)$ of $B$ with $\psi_{1}, \ldots, \psi_{n}$ all distinct. Then $\pi \cong$ $\operatorname{Ind}_{B \dagger G} \psi$.

Proof. It will be enough to show that the matrix coefficient $\varphi: g \mapsto$ $\langle\pi(g) \xi, \xi\rangle$ of $\pi$ coincides with an "obvious" matrix coefficient of the principalseries representation. By Bruhat decomposition, $G=\cup_{w \in W} B w B$, so we can check this on each double coset separately. But for $b_{1}, b_{2} \in B$,

$$
\begin{aligned}
\varphi\left(b_{1} w b_{2}\right) & =\left\langle\pi\left(b_{1} w b_{2}\right) \xi, \xi\right\rangle \\
& =\left\langle\pi(w) \pi\left(b_{2}\right) \xi, \pi\left(b_{1}^{-1}\right) \xi\right\rangle \\
& =\left\langle\pi(w) \psi\left(b_{2}\right) \xi, \psi\left(b_{1}^{-1}\right) \xi\right\rangle \quad \text { (by the eigenvector property) } \\
& =\psi\left(b_{2}\right) \psi\left(b_{1}\right) \varphi(w) .
\end{aligned}
$$

Thus $\varphi$ is determined by its restriction to $W$.

Now if $w \in W$ and $a \in A$,

$$
\begin{aligned}
\psi(a) \varphi(w) & =\langle\pi(w a) \xi, \xi\rangle \\
& =\left\langle\pi\left(w a w^{-1}\right) \pi(w) \xi, \xi\right\rangle \\
& =\left\langle\pi(w) \xi, \pi\left(w a^{-1} w^{-1}\right) \xi\right\rangle \\
& =\overline{\psi\left(w a^{-1} w^{-1}\right) \varphi(w)} \\
& =\psi\left(w a w^{-1}\right) \varphi(w),
\end{aligned}
$$


hence if $\varphi(w) \neq 0, \psi$ must be $w$-invariant. But by assumption, the character $\psi$ is not fixed by any $w \neq 1$ in $W$. Thus $\varphi$ vanishes on $B w B$ for all $w \in W-\{1\}$, hence $\varphi$ is supported on $B$ and $\pi$ is induced from $\psi$ on $B$.

Now we are ready for the main results. It is convenient to begin with Theorem 3.

Proof of Theorem 3. Let $\psi \in\left(K^{\times}\right)^{\wedge}$ and let $\psi_{1}, \ldots, \psi_{n} \in\left(K^{\times}\right)^{\wedge}$ with $\psi_{1} \cdots \psi_{n}=\psi$. It suffices to show that given finitely many elements $g_{1}, \ldots, g_{r} \in$ $G$, none of which is in $Z$, there is a matrix coefficient of $\operatorname{Ind}_{B \uparrow G}\left(\psi_{1}, \ldots, \psi_{n}\right)$ which vanishes on all of them. But for each $i$, the fixed point set of $g_{i}$ in the flag variety $G / B$ is an algebraic subvariety of positive codimension. Thus there exists $x B \in G / B$ such that $g_{i} x B \neq x B$ for all $i$. Then the matrix coefficient of $\operatorname{Ind}_{B \uparrow G}\left(\psi_{1}, \ldots, \psi_{n}\right)$ defined by $x B$ vanishes on $g_{1}, \ldots, g_{r}$, as required. This shows $\lambda_{\psi}$ is weakly contained in our principal-series representation.

Note, however, that the reverse weak inclusion is trivial: since $B$ is solvable, hence amenable, $\left(\psi_{1}, \ldots, \psi_{n}\right)$ is weakly contained in $\operatorname{Ind}_{Z \nmid B} \psi$, and thus by induction in stages, $\operatorname{Ind}_{B \dagger G}\left(\psi_{1}, \ldots, \psi_{n}\right)$ is weakly contained in $\operatorname{Ind}_{B \nmid G} \operatorname{Ind}_{Z \nmid B} \psi \cong \lambda_{\psi}$.

Proof of Theorem 2. We begin by observing that it is enough to prove the theorem when $K$ is countable. The reason is the following. Suppose we know the theorem in this case, and suppose $\pi$ is a unitary representation of $G=\mathrm{GL}(n, K)$ (for general $K$ now) with some central character, say $\varphi \in\left(K^{\times}\right)^{\wedge}$. Because of Theorem 3, we need to show that if $\pi$ is non-trivial on $\operatorname{SL}(n, K)$, then $\pi$ weakly contains $\lambda_{\varphi}$. In other words, we need to show that given $g_{1}, \ldots, g_{r} \in G$, there are matrix coefficients of multiples of $\pi$ converging on these finitely many elements to the canonical matrix coefficient of $\lambda_{\varphi}$ (which is $\varphi$ on $Z$ and 0 off $Z$ ). By adding another $g_{i}$ if necessary, we may assume that $g_{r} \in \mathrm{SL}(n, K)$ and that $\pi\left(g_{r}\right)$ is not the identity operator. Then the subfield $L$ of $K$ generated by the matrix entries of $g_{1}, \ldots, g_{r}$ is countable, and $\left.\pi\right|_{\mathrm{S} L(n, L)}$ is non-trivial, so the desired result follows from the theorem for $L$. (Should $L$ turn out to be finite, first one should replace it by a countably infinite extension field.)

Now we may suppose $K$ is countable. Let $\pi$ be a unitary representation of $G$, say with central character $\varphi$, which is non-trivial on $\operatorname{SL}(n, K)$. Consider the subgroup $Q=H \ltimes U$ of $G$, where $H \cong \mathrm{GL}(n-1, K)$ and $U \cong K^{n-1}$, consisting of matrices of the form 


$$
\left(\begin{array}{c|c}
A & x \\
\hline 0 & 1
\end{array}\right), \quad A \in \mathrm{GL}(n-1, K), \quad x \in K^{n-1} .
$$

Observe first that $\left.\pi\right|_{U}$ must be non-trivial, for any elementary matrix in $G$ can be conjugated into $U$ by an element of $W \subset G$, and thus if $\pi$ were trivial on $U$, it would be trivial on the subgroup of $G$ generated by the elementary matrices, which is all of $\operatorname{SL}(n, K)$ by [7, Lemma 1, p. 376], a contradiction. Thus by Proposition $7,\left.\pi\right|_{U}$ weakly contains the orbit of some $\psi \in \hat{U}$ with trivial stabilizer in $H$. By Lemma 6 , with $G$ in place of $L$ and $Q$ in place of $M, \pi$ weakly contains a representation $\sigma$ of $G$ whose restriction to $Q$ contains an irreducible representation $\rho$ of $Q$ supported on the orbit of $\psi$. By the Mackey machine for $Q, \rho \cong \operatorname{Ind}_{U \uparrow Q} \psi$. In particular, $\left.\rho\right|_{H}$ is equivalent to the regular representation of $H$, and thus $\rho$ remains unchanged up to weak equivalence after tensoring with any representation of $H$ (extended to be trivial on $U$ ). However, $\left.\rho\right|_{U}$ weakly contains the trivial representation of $U$ by Proposition 7 (i) again, so by Lemma 6, $\rho$ weakly contains a representation of $Q$ trivial on $U$, in fact (since we can tensor) any such representation. By Lemma 6, $\sigma$ weakly contains a representation whose restriction to $Q$ contains the regular representtion of $H$, extended to be trivial on $U$. In particular, $\sigma$ restricted to $B(n-1) \ltimes U$, where $B(n-1)$ is the upper-triangular subgroup of $H$, weakly contains every one-dimensional representation of this group, and $\sigma$ restricted to $B=B(n-1) \cdot U \cdot Z$ weakly contains every one-dimensional representation with central character $\varphi$. Since $K$ is infinite, we can choose such a character $\delta=\left(\delta_{1}, \ldots, \delta_{n}\right)$ with $\delta_{1} \cdots \delta_{n}=\varphi$ and with the $\delta_{i}$ 's all distinct. By Lemma 6 again, $\sigma$ weakly contains a representation with a $B$-eigenvector with eigenvalue $\delta$. Then by Lemma $8, \sigma$ (and thus $\pi$ ) weakly contains $\operatorname{Ind}_{B \dagger G} \delta$.

In order to prove Theorem 4 , we need only one more ingredient, namely, to determine when $G$ is and is not amenable.

Proposition 9. $\mathrm{GL}(n, K)$, with the discrete topology and with $n \geqq 2$, is amenable if and only if $K$ is an algebraic extension of a finite field.

Proof. If $K$ is an algebraic extension of a finite field, then $K$ is the union of an ascending sequence $\left\{K_{k}\right\}$ of finite fields. Hence $\mathrm{GL}(n, K)=\underline{\lim } \mathrm{GL}\left(n, K_{k}\right)$ is an inductive limit of finite groups, and so is locally finite and amenable. Note that in this case, $C^{*}(G)$ is an $A F$-algebra (an inductive limit of finite-dimensional $C^{*}$-algebras). 
Therefore we may suppose either that $K \supseteq \mathrm{F}_{p}$ but that $K$ is not algebraic over the prime field, or else that $K \supseteq \mathbf{Q}$.

In the first case, $K$ obviously includes $\mathbf{F}_{p}(t)$, a purely transcendental extension of transcendence degree 1 over $F_{p}$. To show $G=\mathrm{GL}(n, K)$ is nonamenable, it suffices to show that it contains a non-amenable subgroup (since for discrete groups amenability descends to subgroups). Thus we need show only that $\mathrm{GL}(2, \mathbf{Q})$ and that $\mathrm{GL}\left(2, \mathrm{~F}_{p}(t)\right)$ are non-amenable. However, these discrete groups embed densely in $\mathrm{GL}(2, \mathbf{R})$ and $\mathrm{GL}\left(2, \mathbf{F}_{p}[[t]]\left[t^{-1}\right]\right)$, respectively, which are non-amenable Lie groups over local fields. (The non-amenability can be read off very explicitly from the representation theory, say from existence of complementary series.) If any of $\mathrm{GL}(2, \mathbf{Q})$ or $\mathrm{GL}\left(2, \mathbf{F}_{p}(t)\right)$ were amenable, then the Lie group in which it embeds would have a mean invariant under the dense subgroup, and thus under the whole group by continuity, which is a contradiction. So all of these groups are non-amenable.

Proof of Theorem 4. We have seen now that any irreducible representation of $C^{*}(G, \psi)$ weakly contains either a one-dimensional representation or a representation of the principal series. Furthermore, all of the latter (with central character $\psi$ ) are weakly equivalent to $\lambda_{\psi}$. Thus the ideals of codimension 1 (if any) and $\operatorname{ker} \lambda_{\psi}$ are the only possible maximal ideals of $C^{*}(G, \psi)$. Also, it is clear that the set of one-dimensional representations cannot weakly contain $\lambda_{\psi}$, whereas the reverse weak containment holds if and only if $G$ is amenable, or by Proposition 9, if and only if $K$ is algebraic over a finite field. Thus $C_{r}^{*}(G, \psi) \cong C^{*}(G, \psi) /$ ker $\lambda_{\psi}$ fails to be simple only when $K$ is algebraic over a finite field and when there exist one-dimensional representations with central character $\psi$. Finally, when $G$ is amenable, we know that $\lambda_{\psi}$ is faithful on $C^{*}(G, \psi)$.

\section{Extension of the results to other groups}

The reader will note that we have used very little of the structure of GL(n) except for Bruhat decomposition. This immediately suggests that most of our results should go over to other split reductive algebraic groups over $K$. Unfortunately, there is a difficulty in working with $\mathrm{SL}(n)$ or $\mathrm{Sp}(n)$, which can be seen if one tries to prove the analogue of Proposition 7 for the Borel subgroup of $\operatorname{SL}(2, K)$. This group again has the structure $K^{\times} \ltimes K$, but an element $a \in K^{*}$ acts on $K$ by multiplication by $a^{2}$, and unless every element of $K$ is a square (i.e., $K$ has no quadratic extensions if char $K \neq 2$, or $K$ is perfect if char $K=2$ ), the proof of Proposition 7 breaks down. 
However, it turns out our entire theory goes through for the group $G=$ $\mathrm{GSp}(2 n, K)$. This is the subgroup of matrices $a \in \mathrm{GL}(2 n, K)$ satisfying

$$
a J^{t} a=c J, \quad \text { for some } c \in K^{\times} \text {(depending on } a \text { ), }
$$

where $J$ is the standard symplectic matrix

$$
J=\left(\begin{array}{cc}
0_{n} & 1_{n} \\
-1_{n} & 0_{n}
\end{array}\right) \in \mathrm{GL}(2 n, K) .
$$

Note that $\operatorname{GSp}(2, K)=\mathrm{GL}(2, K)$, but that $G$ is a proper subgroup of $\operatorname{GL}(2 n)$ for $n>1$. The map $a \mapsto c$, where $c$ is as in (**), induces an identification of $G_{a b}$ with $K^{\times}$, and the commutator subgroup of $G$ is $\operatorname{Sp}(2 n, K)$. Furthermore, $\mathrm{Sp}(2 n, K)$ is generated by symplectic transvections conjugate to matrices of the form

$$
\left(\begin{array}{cc}
1_{n} & e_{11} \\
0 & 1_{n}
\end{array}\right)
$$

where $e_{11}$ is the usual $(n \times n)$ matrix unit [7,pp. 391-397]. The center $Z$ of $G$ is the same as the center of $\operatorname{GL}(2 n, K)$, and can thus be identified with $K^{\times}$ (cf. Lemma 2 in [7, p. 393]).

Note that if $\varphi \in\left(K^{\times}\right)^{\wedge}$, the one-dimensional representation

$$
a \mapsto \varphi(c) \quad(c \text { as in }(* *))
$$

has central character $\varphi^{2}$, and all one-dimensional representations are of this form. Thus for $\psi \in\left(K^{\times}\right)^{\wedge}$, there exist one-dimensional representations with central character $\psi$ if and only if $\psi(-1)=1$. Except for this change, Theorem 4 goes over verbatim to our new setting.

We sketch now the modifications needed to adapt the proofs for GL $(n)$ to $\operatorname{GSp}(2 n)$. The role of $Q$ in the proof of Theorem 2 will now be played by the parabolic subgroup $Q=H \ltimes U$, where

$$
U=\left\{\left(\begin{array}{c|c}
1_{n} & s \\
\hline 0 & 1_{n}
\end{array}\right): s={ }^{t} s \in M(n, K)\right\}
$$

is unipotent and

$$
H=\left\{\left(\begin{array}{c|c}
z a & 0 \\
\hline 0 & { }^{\prime} a^{-1}
\end{array}\right): a \in \mathrm{GL}(n, K), z \in K^{\times}\right\}
$$

is reductive. $B$ will denote the Borel subgroup $T \ltimes U$, where $T$ is the subgroup 
of $H$ consisting of matrices for which the $a \in \mathrm{GL}(n, K)$ is upper-triangular. Any one-dimensional representation of $B$ factors through

$$
\begin{aligned}
A & =\left\{\operatorname{diag}\left(z a_{1}, z a_{2}, \ldots, z a_{n}, a_{1}^{-1}, a_{2}^{-1}, \ldots, a_{n}^{-1}\right): z, a_{1}, \ldots, a_{n} \in K^{\times}\right\} \\
& \cong\left(K^{\times}\right)^{n+1} .
\end{aligned}
$$

As before, the principal series consists of representations unitarily induced from one-dimensional unitary characters of $B$, and except for the obvious change in the Weyl group $W$ (now $\cong S_{n} \ltimes\left(Z_{2}\right)^{n}$ ), Proposition 1, Theorem 3, and Proposition 9 go through as before. In Lemma 8, the condition that $\psi_{1}, \ldots, \psi_{n}$ be distinct should be replaced by considering any character of $A$ not fixed by any non-trivial element of $W$, i.e., a character of the form

$$
\operatorname{diag}\left(z a_{1}, \ldots, z a_{n}, a_{1}^{-1}, \ldots, a_{n}^{-1}\right) \mapsto \psi_{0}(z) \psi_{1}\left(a_{1}\right) \cdots \psi_{n}\left(a_{n}\right)
$$

with $\psi_{1}, \bar{\psi}_{1}, \ldots, \psi_{n}, \bar{\psi}_{n}$ all distinct.

In the statement of Theorem 2 , of course $\operatorname{SL}(n, K)$ should be replaced by $\operatorname{Sp}(2 n, K)$. The fact that this subgroup is generated by symplectic transvections will guarantee that if $\pi$ is a representation of $G$ non-trivial on $\operatorname{Sp}(2 n, K)$, then $\left.\pi\right|_{U}$ is non-trivial. Thus we need an analysis of the actions of $H$ and $T$ on $\hat{U}$ to replace Proposition 7. For this, note that $U$ may be identified with the additive group of the vector space $\operatorname{Sym}_{n}(K)$ of $n \times n$ symmetric matrices, and each element of $\hat{U}$ has $n(n+1) / 2$ "coordinates" in $\hat{K}$. Since $\operatorname{diag}(z, \ldots, z, 1, \ldots, 1)$ acts on $\operatorname{Sym}_{n}(K)$ by multiplication by $z$, our previous Proposition 7 (applied to the action of $K^{\times}$on each coordinate in turn) shows that every $T$-orbit in $\hat{U}$ has the trivial representation in its closure. Furthermore, a little linear algebra shows that every $H$-orbit in $\hat{U}$ contains a point whose stabilizer in $A$ is contained in the diagonal matrices with all entries \pm 1 . Reasoning as before shows that $\pi$ weakly contains a representation $\rho$ whose restriction to $B$ contains an irreducible representation trivial on $U$, which we can adjust by tensoring with any character of $A$ trivial on the diagonal matrices with entries \pm 1 (extended to be trivial on the commutator subgroup of $T$ ). By another application of Lemma $6, \rho$ weakly contains a representation with a $B$ eigenvector satisfying the condition of Lemma 8. Hence $\rho$ (and thus $\pi$ ) weakly contains a representation of the principal series.

It is also of interest to note that our proofs for $\mathrm{GL}(n)$ also go through with very little modification for $\mathrm{SL}(n)$, provided that $n \geqq 3$. To deal with this case, one merely has to modify the definition of $Q$ in the proof of Theorem 2 so that $Q$ consists of matrices of the form 


$$
\left(\begin{array}{c|c}
A & x \\
\hline 0 & (\operatorname{det} A)^{-1}
\end{array}\right), \quad A \in \mathrm{GL}(n-1, K), \quad x \in K^{n-1} .
$$

Then Proposition 7 has to be modified to deal with the action of $H=$ $\mathrm{GL}(n-1, K)$ on $\left(K^{n-1}\right)^{\wedge}$ dual to the action

$$
g \cdot x=(\operatorname{det} g) g x
$$

In part (ii), the words "with trivial stabilizer" should be replaced by "with stabilizer the $n$-th roots of unity." These of course correspond to the center $Z$ of $\operatorname{SL}(n, K)$. In part (i), the idea is that if $\psi=\left(\psi_{1}, \ldots, \psi_{n-1}\right)$, then by the lemma for GL(1), one can choose a net $b_{\alpha} \in K^{\times}$with $\psi_{1}\left(b_{\alpha} x_{1}\right) \rightarrow 1$ for all $x_{1} \in K$. Then by compactness of $\left(K^{\times}\right)^{\wedge}$, one can pass to a subnet and assume $\psi_{2}\left(b_{\alpha}^{-1} x_{2}\right) \rightarrow \psi_{2}^{\prime}\left(x_{1}\right)$ for some fixed $\psi_{2}^{\prime} \in\left(K^{\times}\right)^{\wedge}$ and for all $x_{2} \in K$. Since $g_{\alpha}=\operatorname{diag}\left(b_{\alpha}, b_{\alpha}^{-1}, 1, \ldots, 1\right)$ has determinant 1 , for $x \in K^{n-1}$,

$$
\begin{aligned}
\psi\left(g_{\alpha} \cdot x\right) & =\psi_{1}\left(b_{\alpha} x_{1}\right) \psi_{2}\left(b_{\alpha}^{-1} x_{2}\right) \psi_{3}\left(x_{3}\right) \cdots \psi_{n-1}\left(x_{n-1}\right) \\
& \rightarrow\left(1, \psi_{2}^{\prime}, \psi_{3}, \ldots, \psi_{n-1}\right) \cdot x .
\end{aligned}
$$

Continuing in this way, we can eliminate one coordinate at a time and show that the trivial character lies in the closure of $H \cdot \psi$.

Notes added in proof.

1. The referee has kindly suggested an alternate argument for the proof of Proposition 9, which is to observe that $\mathrm{GL}(2, \mathbf{Q})$ and $\mathrm{GL}\left(2, \mathrm{~F}_{p}(t)\right)$ contain non-commutative free groups (see, e.g., J.-P. Serre, Trees, Springer-Verlag, Berlin, 1980, §II.1.4 and §II.1.6), and hence cannot be amenable.

2. For the relationship of our work to an earlier paper of A. A. Kirillov (Positive definite functions on a group of matrices with elements from a discrete field, Dokl. Akad. Nauk SSSR 162 (1965), 503-505), see a forthcoming paper Un complément à un théorème de Kirillov sur les caractères de $\mathrm{GL}(n)$ d'un corps infini discret of the second author, to appear in C.R. Acad. Sci. Paris, Sér. I Math.

\section{REFERENCES}

1. J. Dixmier, C*-Algebras, North-Holland, Amsterdam, 1977.

2. J. M. G. Fell, The dual spaces of $C^{*}$-algebras, Trans. Am. Math. Soc. 94 (1960), 365-403.

3. P. de la Harpe, Reduced $C^{*}$-algebras of discrete groups which are simple with a unique trace, in Operator Algebras and Their Connections with Topology and Ergodic Theory, Proceedings, Busteni, Romania 1983, Lecture Notes in Math., No. 1132, Springer-Verlag, Berlin, 1985, pp. 230-253. 
4. R. E. Howe, On representations of discrete, finitely generated, torsion-free, nilpotent groups, Pacific J. Math. 73 (1977), 281-305.

5. R. E. Howe and J. Rosenberg, The unitary representation theory of $\mathrm{GL}(2)$ of an infinite discrete field, Abstracts Am. Math. Soc. 7 (1986), No. 2, 199, \#827-22-42.

6. J. E. Humphreys, Linear Algebraic Groups, Graduate Texts in Math., Springer-Jerlag, New York, 1975.

7. N. Jacobson, Basic Algebra I, 2nd ed., Freeman, New York, 1985.

8. D. A. Kazhdan, On the connection of the dual space of a group with the structure of its closed subgroups, Funkcional. Anal. i Priložen. 1 (1967), 71-74.

9. G. W. Mackey, On induced representations of groups, Am. J. Math. 73 (1951), 576-592.

10. E. Thoma, Die unzerlegbaren, positiv-definiten Klassenfunktionen der abzahlbar unendlichen, symmetrischen Gruppe, Math. Z. 85 (1964), 40-61.

11. E. Thoma, Eine Charakterisierung diskreter Gruppen vom Type I, Invent. Math. 6 (1968), 190-196. 